\title{
Causes of kidney disease among patients in a hemodialysis unit
}

\begin{abstract}
It is Estimated That one in ten adults Suffers CKD (chronical kidney dissease) in the world, a result to a progressive Increase in the incidence and prevalence of chronic diseases, Which are able not only to trigger it, but Also to accelerate the progress of it Until the stage V and merit renal replacement therapy. General Objective: To know the different underlying conditions That trigger boxes chronic renal failure leading to renal replacement therapy or hemodialysis Patients in a private center of Valencia-Carabobo Materials and Methods: A descriptive study With field design, clinical and epidemiological and transitional was Conducted by reviewing 86 clinical records, records of current: such as medical data of all ADMITTED Patients in hemodialysis units of the Clinical Center in the 2015 Results: history of hypertension in $92 \%, 53 \%$ DM, ITU $30 \% .52 .3 \%$ are over 60 years Patients. Conclusion: Among the underlying conditions are more closely related That to the development of ERC With entrance to hemodialysis unit of a private medical center find the development of nephrosclerosis, followed by diabetes mellitus for the development of diabetic nephropathy and other diseases kidney thirdly, mainly associated pre-existing With anatomical malformations, losing relevance recurrent urinary tract infections.
\end{abstract}

Keywords: ERC, hemodialysis, nephroangiosclerosis, diabetes mellitus, clinical history, hemodialysis, peritoneal dialysis and kidney transplantation
Volume 7 Issue 5 - 2019

Patricia Perez, Jesus Perez, Perez Kengely,
Julio Reyes, Romero N
Faculty of Health Sciences, University of Carabobo,Venezuela

Correspondence: Romero N, Faculty of Health Sciences, University of Carabobo, School of Medicine Valencia, Venezuela, Tel +34 69243 1996, Email neyduelizabeth@gmail.com

Received: September 09, 2019 | Published: October 18, 2019
Abbreviations: CKD, chronical kidney dissease; ASN, American society of nephrology; EPIRCE, Epidemiology study of chronic kidney disease in Spain; PMP, per million population; DM, diabetes mellitus

\section{Introduction}

Chronic kidney disease (CKD) is the result of progressive and irreversible loss of renal function resulting from parenchymal destruction observed in the course of various diseases that affect the kidneys and other related structures currently system renal. ${ }^{1} \mathrm{He}$ considers this disease as an epidemic that affects more than $10 \%$ of the world population, estimated about 720 million patients affected aproximadamente. ${ }^{2}$ According to the American Society of Nephrology $(\mathrm{ASN})$, it is estimated that for every ten adults in the world, at least one suffers from kidney disease, without distinction between developed and underdeveloped countries. As a result in imminent and progressive increase in the incidence and prevalence of chronic diseases such as hypertension and are diabetes mellitus. Which are able not only to trigger it, but also to accelerate the progress of it until the stage $\mathrm{V}$ and merit renal replacement therapy (hemodialysis). ${ }^{3}$ These triggers can vary in frequency of one or the other depending on both geographic location or economic class of each country. It can also be associated with other less frequent triggers etiologies of CKD as urinary tract infections, renal trauma, adverse effects associated with drugs and drugs, surgical procedures, congenital malformations or unmodifiable such as age, race and gender processes. Black patients the most vulnerable, like those over 60 years with a predominance towards gender femenino. ${ }^{4}$

The main risk factors associated with possible development of CKD have changed over time. Glomerulonephritis previously was considered the most common cause of kidney disease, however, that today is considered diabetic nephropathy has come to occupy the first place, especially in developed countries. Followed by hypertensive nephrosclerosis, positioning glomerulonephritis place. ${ }^{5}$ The third possible explanation for these changes lies in the constant and proportional rise of diabetes mellitus, making it a disease pandémica. ${ }^{6}$ On the other hand, advances in the proper management glomerulonephritis are preventing the disease becomes chronic so, its importance in the genesis of renal disease has been disminuyendo.? According to the Chilean Society of Nephrology Diabetes is the leading cause of chronic kidney disease in most countries of the world. Despite the obvious progress in the understanding and management of the variables that influence the development of diabetic nephropathy, it has shown a significant increase in the prevalence of chronic kidney disease in diabetic patients, whose cause has not been determined. Moreover, patients with diabetic nephropathy have a much higher morbidity and mortality in patients with renal disease from other causes, which is attributed to comorbidity and partly to factors of diabetes. ${ }^{6}$

The prevalence of diabetes in developed countries such as Spain is estimated close to $14 \%$ of the adult population. In addition, the ERC is an emerging problem worldwide. The results of Epidemiology study of chronic kidney disease in Spain (EPIRCE) show that about $10 \%$ of the adult population suffer from some degree of CKD. It is anticipated that $27.9 \%$ of patients with DM2 have CKD in Spain and more than $35 \%$ had microalbuminuria, proteinuria or ERC. ${ }^{8}$ Meanwhile a study of cross-cutting and metacentric in Buenos Aires, Argentina concludes that the primary cause of CKD was the HTA through nephroangiosclerosis, leaving the DM as a second trigger. This study was conducted in different centers and nephrology clinics, separated into two stages. The first stage in 2010, in which patients from different 
centers of the City of Buenos Aires and the second stage, which took place during the years 2011 and 2012, in which patients City joined included Buenos Aires, the Buenos Aires Province and the Province of Santa Fe. A total of 611 patients, $46.5 \%$ women and $53.0 \%$ men were included. The mean age was $71.5 \pm 14.0$ years and more than $50 \%$ of the population had more than two years of diagnosis. ${ }^{9}$

In Latin America there is also a trend towards a progressive increase in renal replacement therapy. The average prevalence of all three modalities of treatment increased from 119 patients per million population (PMP) in 1991 and has become 199 and 221 in 2002 and 2003 respectively. The incidence has also increased significantly, from 27.8 in 1992 to $94 \mathrm{pmp}$ in the year $2002 .{ }^{10}$ The ERC becomes a public health problem of the first order given not only to associated comorbidities, but also the economic cost and impact on the patient's lifestyle involving replacement therapies. This situation represents a socio-health and economic problem of considerable magnitude, not only individually, but also for nación. ${ }^{11}$ It is believed that individuals of low socioeconomic status are more likely to develop the disease. According to a study in Valencia, Carabobo in 2011 where they compared the levels of depression, anxiety and stress in nephropathy patients undergoing peritoneal dialysis and hemodialysis in relation to socioeconomic status. It was concluded that $46.7 \%$ of the sample belonged to a Graffar IV, equivalent to socioeconomic status bajo. ${ }^{12}$ In Venezuela currently ERC casuistry about 19,000 patients are estimated. Of these only about 14,000 are on dialysis, patients belonging to the state 5,400 Carabobo. ${ }^{11}$ while the corresponding replacement therapy (hemodialysis, peritoneal dialysis and kidney transplantation) epidemiological data is accurately known, the information corresponding to the specific causes of these in our country are largely unknown since about 2004 .

Encouraged to promote prevention as the fundamental medicine in our society decided to explore one of the diseases most prevalent in the world population affecting patients of all ages for different reasons, why we propose to know the different underlying pathologies triggering boxes chronic kidney disease (stage V), leading to the hemodialysis renal replacement therapy in patients in a private center Valencia Carabobo state. In another area this research fits great interest for that alien individual to health personnel, this group mainly made up of the same patients suffering from this clinical picture as well as their relatives being, providing accurate relevant and updated information about factors that triggered and regional level statistical correlation. It is also expected that this project will motivate the realization of future similar research in various centers around the country and reach have a national epidemiological control of the etiology of the disease, comparable with existing records in other countries of the continent and the world. In this paper seeks to fill this gap slightly concerning risk factors of CKD stage triggers $\mathrm{V}$ with hemodialysis income epidemiological information. Presenting the results of a study that maintains a descriptive level with a field design, clinical and epidemiological and cross-sectional, conducted by reviewing medical records, including records of medical data present in all patients admitted to hemodialysis therapy in the unit of a private center of Valencia, Edo Carabobo.

\section{Material and methods}

It is a descriptive observational research with a cross-sectional and retrospective design. The study population was represented by all those patients who are on renal replacement therapy, have been in such treatment in the hemodialysis unit in the city of Valencia,
Carabobo state between the months of January-December 2015. The sample type probabilistic no census, made up of the medical records of 86 patients met the condition set. Once the necessary permission as the institution case study and the hemodialysis unit recorded history was obtained according to the variables included in the research were described. The data collection was conducted by reviewing medical records, the logbook outpatient and the book of the hemodialysis, in order to meet the different underlying conditions that trigger boxes chronic kidney disease stage $\mathrm{V}$ entering the unit. The results were organized in tables frequency distribution (absolute and relative) to determine the frequency with which each variable for the admission of patients presented, allowing to determine the most common and such categorizing form causes of importance.

\section{Results}

In this it corresponds to a $52.33 \%$ age of the patients were over 60 years ( 45 cases), followed by those with 36 to 59 years $(39.53 \%=34$ cases). As for the male sex she predominated with $63.95 \%$ (55 cases) over female (31 cases) (Table 1). Regarding socioeconomic level as measured Graffar, most frequent were those of stratum IV and V with similar proportion $(38.37 \%$ a like $=33$ cases), while $23.26 \%$ of patients were Stratum III (20 cases) (Table 2 ). The predominant history among the patients studied was the presence of hypertension $(91.86 \%=79$ cases), the most frequent antecedent in both sexes: male female $(29 / 31)$ and (50/55). The second most frequent antecedent was the type 2 diabetes mellitus $(53.49 \%=46$ cases) more frequent in women $(20 / 31)$. Patients with chronic kidney disease who had a history of anatomical malformations most frequent were those with the presence of renal stones (10 cases), followed by those with bilateral polycystic ( 9 cases), thirdly, they presented those pathologies like tumors (5 cases) (Table 3).

Table I Chronic kidney disease cases of distributed by age and sex. Hemodialysis. Valencia, 2015

\begin{tabular}{lll}
\hline Ages & F & $\%$ \\
\hline I8-35 & 7 & 8.14 \\
$36-59$ & 3.4 & 39.53 \\
$>60$ & 45 & 52.33 \\
Sex & F & $\%$ \\
Female & 31 & 36.05 \\
Male & 55 & 63.95 \\
Socioeconomic & F & $\%$ \\
III & 20 & 23.26 \\
IV & 33 & 38.37 \\
V & 33 & 38.37 \\
Total & 86 & 100 \\
\hline
\end{tabular}

Source: Own Investigation Data ${ }^{12}$ 
Table 2 Distribution history of hypertension, DM E ITU according to sex. Hemodialysis Valencia, 2015

\begin{tabular}{lllllll}
\hline Sex & Female & & Male & \multicolumn{3}{l}{ Total } \\
\hline & $(\mathbf{N}=\mathbf{3 1})$ & & $\mathbf{( N = 5 5 )}$ & & $\mathbf{( N = 8 6 )}$ \\
\hline Background & $\mathrm{F}$ & $\%$ & $\mathrm{~F}$ & $\%$ & $\mathrm{~F}$ & $\%$ \\
HTA & 29 & 93.55 & 50 & 90.91 & 79 & 91.86 \\
DM & 20 & 64.52 & 26 & 47.27 & 46 & 53.49 \\
ITU & 10 & 32.26 & 16 & 29.09 & 26 & 30.23 \\
$\begin{array}{l}\text { Congenital } \\
\text { malformations }\end{array}$ & 10 & 32.26 & 22 & 40 & 32 & 37.21 \\
\hline
\end{tabular}

Source: Own Investigation Data ${ }^{12}$

Table 3 Analysis of most frequent type of defects in patients Anatomic study. Hemodialysis. Valencia, 2015

\begin{tabular}{lll}
\hline Anomaly & F & $\%$ \\
\hline Nephrolithiasis & 10 & 31.25 \\
bilateral polycystosis & 9 & 28.12 \\
LOE & 5 & 15.63 \\
Obstructive uropathy & 3 & 9.38 \\
Congenital single kidney & 3 & 9.38 \\
Toxic nephropathy & one & 3,13 \\
Transplant rejection & one & 3,13 \\
Total & 32 & 100 \\
\hline
\end{tabular}

Source: Own Investigation Data ${ }^{12}$

\section{Discussion}

This research is based on determining the causes of ERC to progress to stage $\mathrm{V}$ renal therapy involving replacement need. It found that two-thirds of the study population were male compared to female representing a third party, similar to the work done in Argentina by M. Lombardo between 2010-2012.9 Reaffirming values that men are more likely to develop CKD progresses to stage $\mathrm{V}$ on hemodialysis. Predominating in patients with older age at 60 years, followed by the group between 36-59años respectively, and a minimum presence of adults between 18 and 35, noting that the risk of progression of $\mathrm{CKD}$ to stage $\mathrm{V}$ increases at ages more advanced. Regarding the level socioeconomic valued by Graffar scale more frequently reported in the socioeconomic IV and V with low incidence between layers II and III respectively, coinciding with the result Rubio $\mathrm{R}$ et al. ${ }^{12}$ between 2010- 2011In Valencia Carabobo12 state where the predominant Graffar was the level IV.

Once again it shows that hypertension is a key risk factor in the onset of CKD patients being more susceptible older age and male and resulted in a study presented by Lombardo M. ${ }^{9}$ More than half of patients who are in the process of hemodialysis presents Diabetes Mellitus (DM) totally contrary to the results reported in a study by Gómez-Huelgas R et al in the year $2014 .{ }^{8}$ where only one-third of the study population $\mathrm{He}$ is carrying this pathology. In the study sample the incidence of female patients with a history of Diabetes Mellitus is lower as the male but still there is a predominance in (over 60 years) advanced ages finding consistent with those obtained by Maria $\mathrm{S}$ and collaborators in Chile in the year $2008 .{ }^{6} \mathrm{~A}$ history of urinary tract infectionin patients admitted to the center, it was absent found in $70 \%$ of the population, considering it is not a significant risk factor predisposing to the disease. It is valid to note that they are men with CKD who mostly have such history compared to women. Important fact since by female anatomical factors are more likely to get infections of this type. However it was determined that the incidence of pre-existing anatomical malformations was a history of major relevance in male compared with female and were a predisposing factor for the development of the disease in ages tempranas. ${ }^{7}$ It is noteworthy that a patient with chronic kidney disease may have one or more risk factors, so the onset and progression of the disease to stage $\mathrm{V}$ can be multifactorial and that the potential risk of disease progression increases directly and proportionately to the presence of more predisposing factors.

\section{Conclusion}

After performing this research and exhaustive of the results of the same analysis it can be concluded that among the underlying conditions that are more closely related to the development of chronic kidney disease entering hemodialysis unit private center valence, Carabobo are in first instance hypertension, specifically for developing nephrosclerosis present in $92 \%$ of patients studied. Diabetes mellitus for the development of diabetic nephropathy is present in 53\% of the sample and thirdly the other renal diseases, primarily associated with existing anatomical malformations. Becomes less relevant infections recurrent urinary tract, with only $30 \%$ incidence. Similarly it could be concluded that the age group with the highest incidence was for patients over 60 years, representing $52 \%$ of the study population, followed by those aged between 36 and $59,40 \%$ and finally those patients under 36 years with only $8 \%$. These results can be linked to degeneration of the renal parenchyma own involving age correlated with pre-existing conditions already mentioned and progress. Like wise it was determined that the cases of young patients admitted to the center were mainly due to other proper kidney entities, not necessarily related to hypertension or diabetes mellitus, such as are some preexisting anatomical alteration (kidney patients, nephrolithiasis, polycystic bilateral, also it found that gender highest incidence for developing CKD male was $63 \%$ vs. $37 \%$ for the female, fulfilling a ratio of 2: 1 . Finally it was evident that socioeconomic status in the center where the research was conducted is a factor that directly affects the development and progress of the clinical entity. Taking this consideration because $76 \%$ of patients were located in grades IV and V of Graffar scale, grade III followed by $23 \%$ and ultimately Graffar grades I and II with $0 \%$. Leading to the conclusion that the lower was the higher socioeconomic status will be the progression of kidney disease stage $\mathrm{V}$, either by lack of access to treatment, not having the resources necessary to fulfill the same or lead lifestyles that represent a risk and impact on the renal parenchyma as is diet.

\section{Recommendations}

I. Urge all hemodialysis centers in the nation with adequate control regarding the registration of all patients admitted to their units.

II. Conduct similar research in various centers around the country and reach have a national epidemiological control of the etiology of the disease, comparable with existing records in other countries of the continent and the world.

III. For future studies consider new variables that may affect the development of ERC and expand knowledge regarding triggers it. 
IV. Conduct studies with large samples in terms of number of patients thus allowing to reach a genuine national consensus as the basis and epidemiological registry.

$\mathrm{V}$. Encourage the creation of public policies that promote the prevention of hypertension and DM2 as pathologies basis for the development of ERC in order to reduce the caseloads of this disease and improve health in our country.

\section{Acknowledgments}

None.

\section{Funding details}

None.

\section{Conflicts of interest}

The author declares there is no conflict of interest.

\section{References}

1. M Prague, Ejido J. Chronic renal failure. In: Arias M Hernando editor Clinical nephrology. 4th edn. Spain: Pan; 2013. p. 799-804.

2. Eckardt KU. The overall issue of kidney disease. The Lancet 2013;382(9987):101.

3. Thomas D Boyer, Bruce Kaplan. Renal failure and liver transplantation. Clinical Liver Disease. 2013;2(S5):95-97.

4. Al Khayat R, Lefevre G. Analytical test-clinical hemodialysis. Acta Latin American clinical biochemistry. 2008;42(4):580.
5. Alberto Martinez-Castelao. Detection and management of CKD. Nephrology. 2014;34(2):243-262.

6. Sanhueza M. Diabetes and Hemodialysis. Rev Med Chile. 2008;136:279286.

7. Graupera I, Cardenas A. Approach Diagnosis of Renal Disease Patients with cirrhosis. Clinical Liver Disease. 2013;2(4):43-45.

8. Gomez-strikes R, Martinez-Castelao A, Artola S, et al. Consensus Document on the treatment of type 2 diabetes in patients with chronic kidney disease. Nephrology Madrid. 2014;34(1):34-45.

9. Lombardo M. Current status of anemia associated with kidney disease in a population sample of patients with impaired renal function without dialysis requirements in Argentina - study APREDIA. Rev Nefrol dial Transpl. 2014;34(3):1-3

10. KR Furaz Czerpak, A Puente García, E Corchete Prats, et al. Successful pregnancy in a patient with chronic renal failure in the hemodialysis program. Nephrology. 2011;31(2):219-221.

11. Linares sambrano JD, Campos TM. Economic impact of renal replacement therapy in the public health expenditure Venezuela. Caracas. Andrés Bello Catholic University Master in Management of health care services. 2010.

12. Rubio R, N Sanchez, Jimenez M, et al. Comparison of levels of depression, anxiety and stress in nephropathy patients undergoing peritoneal dialysis and hemodialysis and their relationship with socioeconomic status. Dr. Enrique Tejera Hospital City (CHET) and outpatient dialysis units. Valencia, Carabobo state. Advances in health sciences. 2012;1(2):14. 